\title{
Nondegenerated cystic neuroendocrine tumor of the pancreas: a case report
}

\author{
Keisuke Noda ${ }^{1 *}$, Tamotsu Kuroki ${ }^{1}$, Mampei Yamashita', Takanori Hirayama ${ }^{1}$, Koji Natsuda ${ }^{1}$, Shinichiro Kobayashi ${ }^{1}$, \\ Takayuki Tokunaga', Kosho Yamanouchi ${ }^{1}$, Hiroaki Takeshita', Shiro Miura ${ }^{2}$ and Shigeto Maeda ${ }^{1}$
}

\begin{abstract}
Background: Pancreatic neuroendocrine tumors (PNETs) are typically solid neoplasms but, in very rare cases, present as cystic lesions. We describe a case of a cystic neuroendocrine tumor that developed as a small cystic lesion.

Case presentation: In 2011, a 66-year-old Japanese woman underwent computed tomography (CT) that revealed a cystic lesion in the tail of the pancreas measuring $9 \mathrm{~mm}$. She did not have any symptoms. She underwent a CT scan every year thereafter. The cystic lesion gradually increased and was $40 \mathrm{~mm}$ in 2019; endoscopic retrograde pancreatography (ERP) was then performed. Cytological examination demonstrated class Illb adenocarcinoma, and we conducted laparoscopic distal pancreatectomy. Pathological examination showed PNET.
\end{abstract}

Conclusion: Although cystic change of PNET is generally caused by ischemia or necrosis inside the tumor, in our case, PNET occurred as a small cyst that increased without changing form.

Keywords: Pancreatic neuroendocrine tumor, Cystic lesion, Pancreas

\section{Background}

Pancreatic neuroendocrine tumors (PNETs) are very rare, accounting for only 1 to $2 \%$ of all pancreatic tumors [1]. PNETs are typically solid neoplasms but present as cystic lesions in very rare cases [2]. In general, the mechanism of cystic degeneration in PNET is ischemia or necrosis inside the tumor because of an increase in tumor diameter or intratumoral hemorrhage due to blood vessel rupture [2].

Herein, we describe a case of a cystic PNET that developed as a small cystic lesion.

\section{Case presentation}

In 2011, a 66-year-old Japanese woman underwent unenhanced computed tomography (CT) that showed an incidental cystic lesion in the tail of the pancreas measuring $9 \mathrm{~mm}$ (Fig. 1a). She did not have any symptoms.

\footnotetext{
* Correspondence: kenoda-ngs@umin.ac.jp

${ }^{1}$ Department of Surgery, National Hospital Organization Nagasaki Medical

Center, 2-1001-1, Kubara, Omura City, Nagasaki 856-8562, Japan

Full list of author information is available at the end of the article
}

After that, she underwent a CT scan every year. Her medical histories were as follows: breast cancer, $\mathrm{HCV}$ carrier, hypertension, and type 2 diabetes mellitus. Laboratory tests, including carcinoembryonic antigen (CEA) and carbohydrate antigen 19-9 (CA19-9), were normal. In 2013, the cystic lesion had grown to $14 \mathrm{~mm}$, and the peripheral rim was enhanced on contrastenhanced CT (CECT) (Fig. 1b). Although the cystic lesion continued to increase gradually, its form did not change. In 2018, the cystic lesion had become $33 \mathrm{~mm}$ and contained fluid at the bottom, which was considered clot on unenhanced CT (Fig. 1c). However, endoscopic ultrasound did not reveal a mural nodule lesion. In 2019, the cystic lesion was $40 \mathrm{~mm}$, and the outer rim was thinner on CECT (Fig. 1d). Endoscopic retrograde pancreatography (ERP) showed that the main pancreatic duct was stenotic without any connection to the cystic lesion (Fig. 2). The main pancreatic duct further exhibited tapered stenosis at the pancreatic body and a slightly dilated main pancreatic duct on the distal side. Cytological examination demonstrated class IIIb 


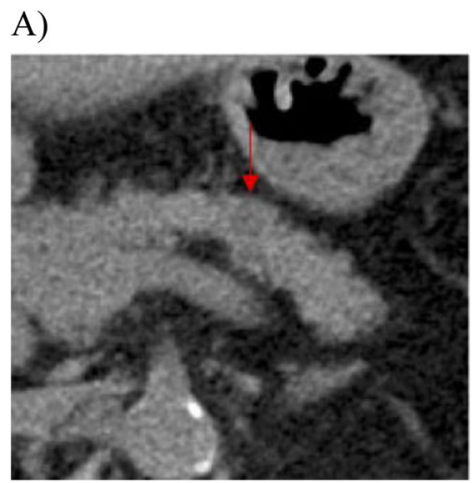

B)

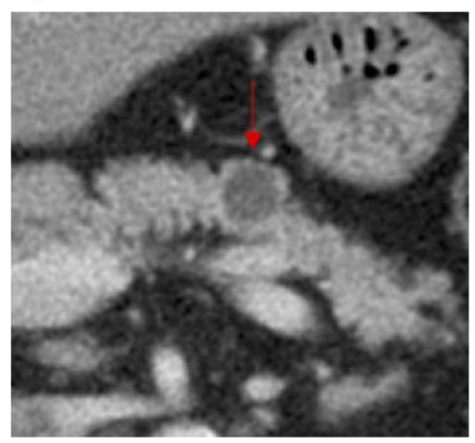

C)

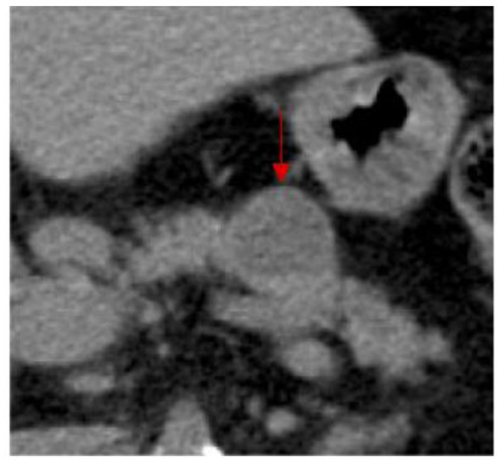

D)

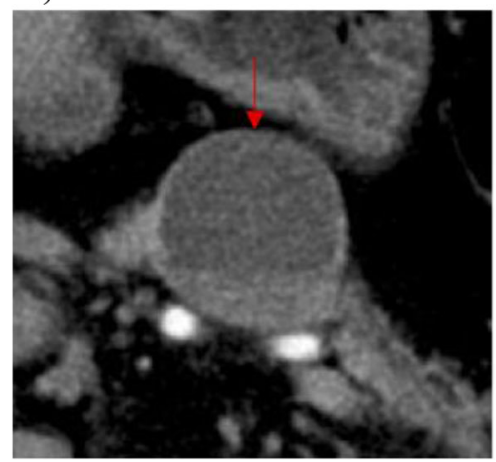

Fig. 1 Abdominal CT showing the cystic lesion in the tail of the pancreas that had become larger without a change in form. a Unenhanced CT showing a 9-mm cystic lesion in 2011. b Contrast-enhanced $C T$ (CECT) showing a 14-mm cystic lesion in 2013. The peripheral rim was enhanced. c Unenhanced CT of a 33-mm cystic lesion and consisting of fluid at the bottom, which was considered clot in 2018. d CECT showing a 40-mm cystic lesion in 2019. The outer rim became thinner

adenocarcinoma (Fig. 3). The patient also underwent magnetic resonance imaging (MRI), demonstrating $\mathrm{T} 1$ low, T2 high, and fluid-fluid levels (Fig. 4). We considered that an intraductal papillary mucinous neoplasm or a mucinous cystic neoplasm was complicated by pancreatic cancer, and we performed laparoscopic distal pancreatectomy. The patient had an uneventful postoperative recovery and left the hospital 12 days later.

Histopathology showed a cystic lesion measuring $21 \times$ $11 \mathrm{~mm}$ in the tail of the pancreas (Fig. 5a). The content

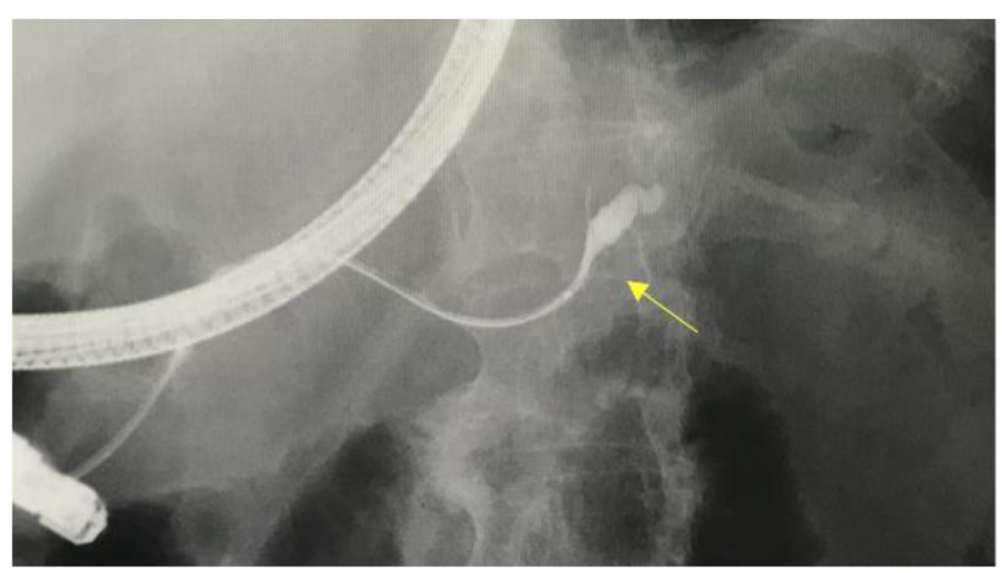

Fig. 2 Endoscopic retrograde pancreatography revealed that the main pancreatic duct was stenotic without any connection to the cystic lesion. The main pancreatic duct showed tapered stenosis at the pancreatic body and a slightly dilated main pancreatic duct on the distal side 


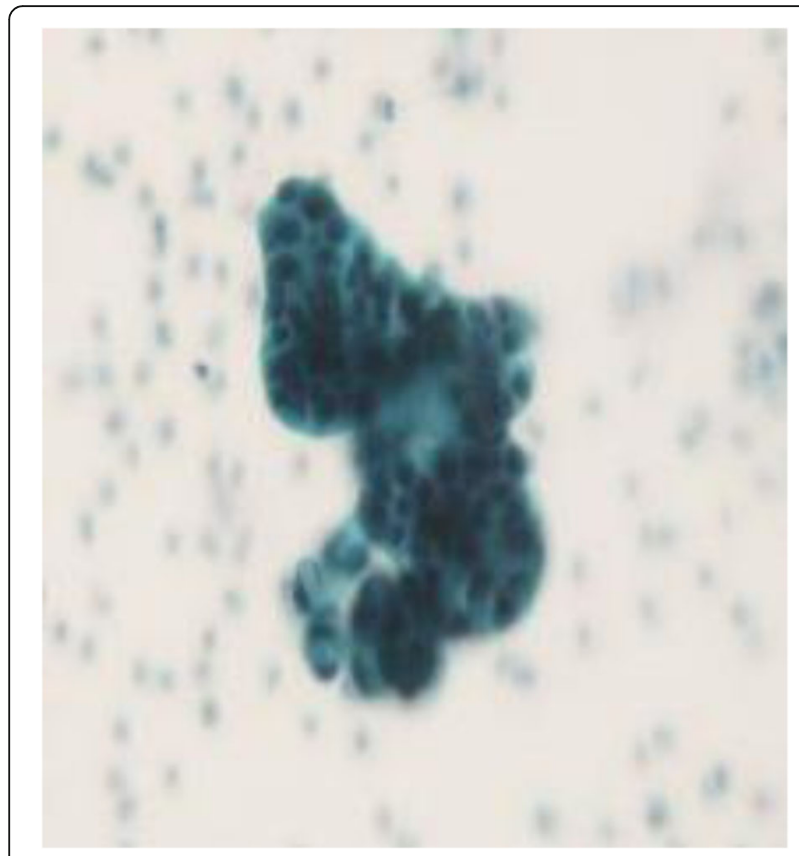

Fig. 3 Abdominal magnetic resonance imaging demonstrating T2 high and a fluid-fluid level

of the cystic lesion was serous fluid without blood. The wall of the cyst was filled with fibrous connective tissue, with some growth of tumor cells consisting of atypical cells with dense chromosomes on the inner surface. There was no necrosis or mucus in the lumen (Fig. 5b). The tumor cells displayed ribbon-like hyperplasia and gland-like structures. There were small blood vessels around the cells and no mitotic figures

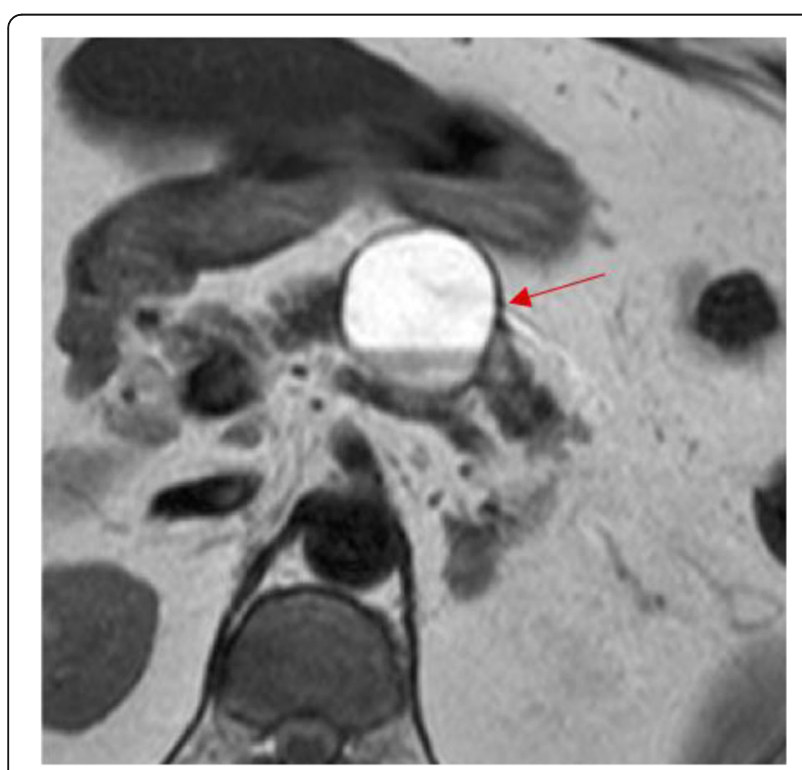

Fig. 4 Cytological examination demonstrated class Illb adenocarcinoma

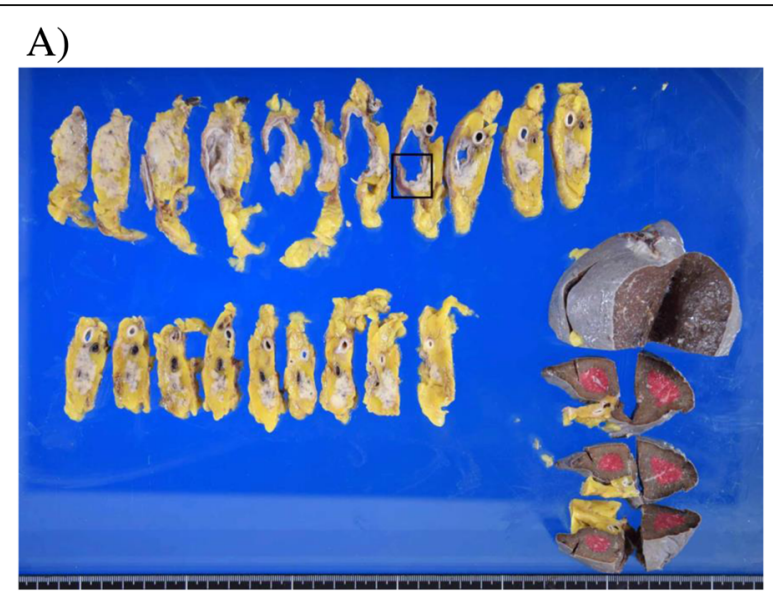

B)

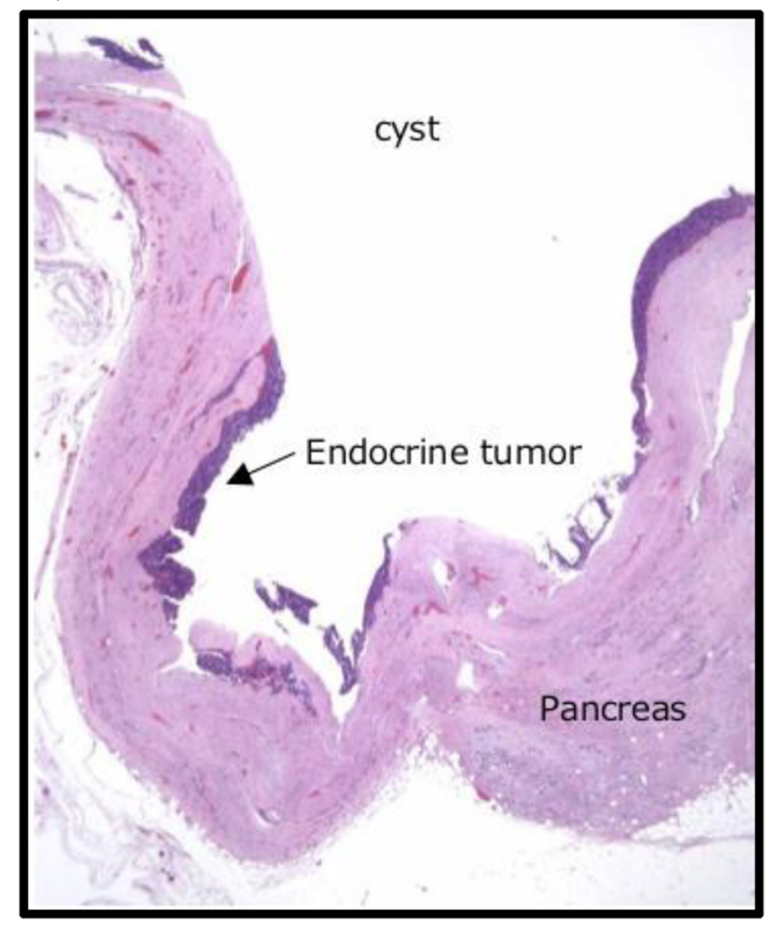

Fig. 5 a Macroscopic findings demonstrated a cystic lesion measuring $21 \times 11 \mathrm{~mm}$ in the tail of the pancreas. $\mathbf{b}$ Histopathology revealed that the wall of the cyst was filled with fibrous connective tissue, with some growth of tumor cells consisting of atypical cells with dense chromosomes on the inner surface (hematoxylin and eosin stain)

at $10 \mathrm{hpf}$ (Fig. 6a). Cytological examination showed positive staining for INSM1 and synaptophysin, which are highly specific markers for neuroendocrine tumors (Fig. $6 \mathrm{~b}$ and c). The Ki-67 index was less than $1 \%$, making this a G1-type tumor. Immunohistochemical markers of insulin and glucagon were negative.

\section{Discussion}

Neuroendocrine tumors account for only $1-2 \%$ of all pancreatic neoplasms, and cystic lesions account for less 


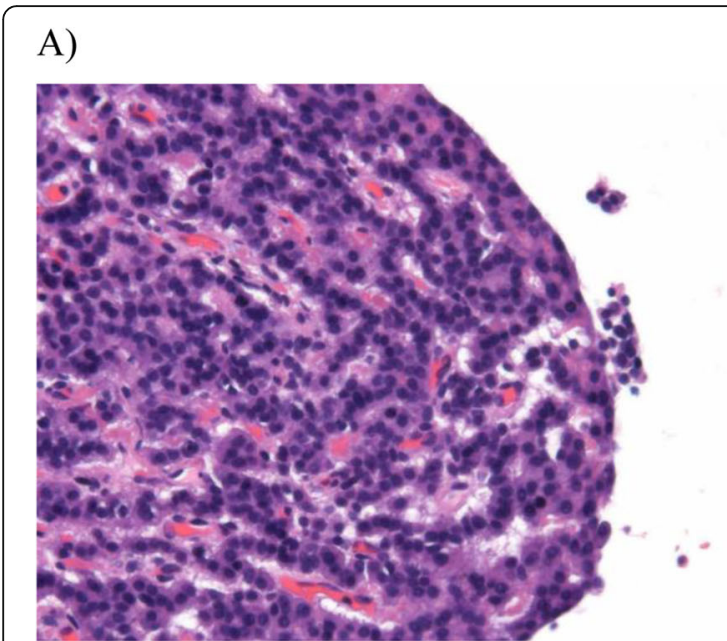

B)

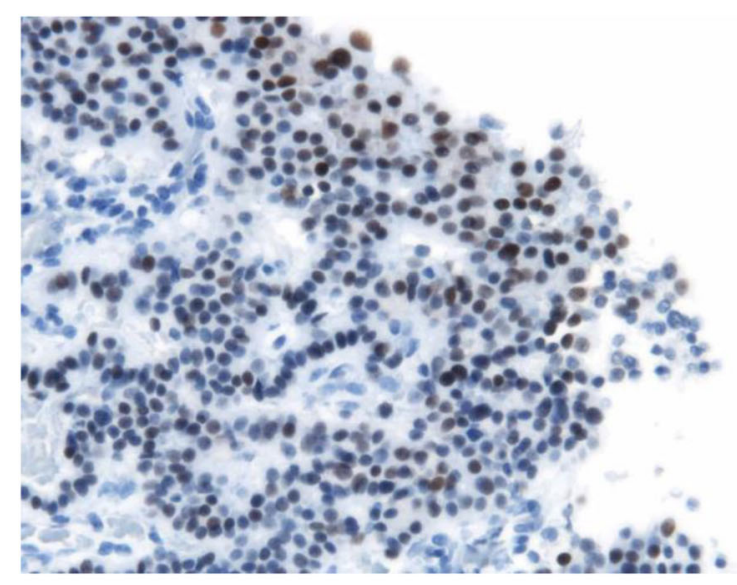

C)

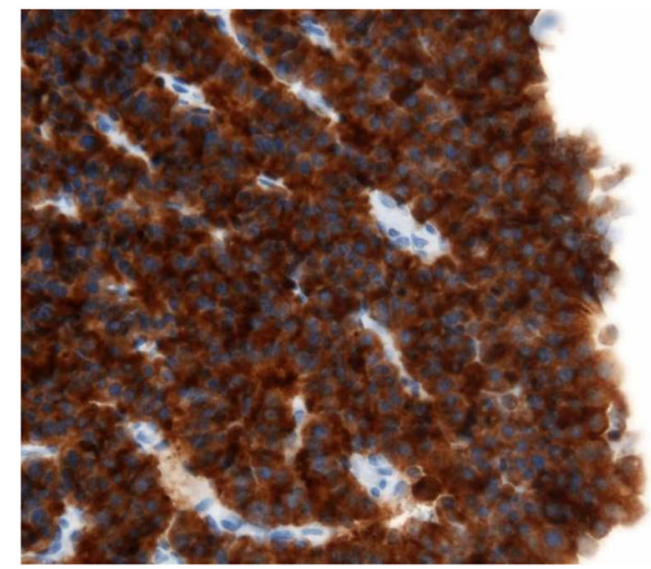

Fig. 6 Histopathological findings for the resected pancreas. a Tumor cells had ribbon-like hyperplasia and gland-like structures. There were small blood vessels around the cells and no mitotic figures at $10 \mathrm{hpf}$ (hematoxylin and eosin staining). $\mathbf{b}$ and $\mathbf{c}$ Tumor cells showed positive results for INSM1 (b) and synaptophysin (c) by immunohistochemical staining than $10 \%$ of all neuroendocrine tumors; thus, cystic neuroendocrine lesions are very rare [1]. However, the increasing number of diagnostic examinations enables more detection of cystic lesions of the pancreas [3]. The mean age at diagnosis is 53 years, and there is no evidence of sex predilection [2]. Overall, cystic PNETs appear to be larger, of a lower grade and present at a lower stage than typical solid PNETs $[4,5]$. In addition, incidences of regional lymph node metastases with cystic PNETs are rare compared with typical solid PNETs [6]. Most cystic PNETs are nonfunctional, and the diagnosis is usually made incidentally or secondary to mass-dependent symptoms such as abdominal pain. Except for neuroendocrine microadenomas, all neuroendocrine tumors are considered to have malignant potential and should be considered for surgical resection $[6,7]$. However, the diagnosis of cystic PNETs with conventional axial imaging, such as CT and MRI, is extremely difficult [8]. In our case, the tumor increased gradually and the cytology for the specimen obtained by ERP-demonstrated indicated class IIIb adenocarcinoma; therefore, we were not able to achieve a preoperative diagnosis of cystic PNET.

With regard to how preoperative ERP cytology showed class IIIb adenocarcinoma, it was presumed that we had taken some PNET cells as specimens that had fallen into the pancreatic duct from the cystic lesion. In addition, these cells were suspected to be adenocarcinoma on cytology. We have determined that the release of some PNET cells from the cystic lesion may have been caused by the guidewire technique or a very small connection that had not been detected by some diagnostic examinations.

We also considered the mechanism of cystic degeneration. There are two main hypotheses regarding the presence of cystic components in PNETs. Some investigators speculate that the fibrous capsules of PNETs hinder blood supply to the lesion, leading to necrosis and subsequent cystic degeneration [9, 10]; others postulate that hemorrhage may occur in PNETs, which are highly vascular, and that cystic degeneration is a consequence of such hemorrhage [11, 12]. Regardless, the precise mechanisms have not been fully elucidated [5].

In our case, the tumor presented as a cystic lesion when it was first detected in 2011, and histopathology showed some growth of tumor cells on the inner surface of the wall of the cyst, with no necrosis or mucus in the lumen. Consequently, it was presumed that the mechanism of cystic degeneration in our case was not necrosis but intralesional hemorrhage before it was detected.

Nevertheless, we also considered another hypothesis: this lesion had occurred as a small cyst that increased as it was. Indeed, it was detected as a very small cystic lesion that increased without a change in form. These findings suggest that our patient's PNET developed as a cyst that did not degenerate. 
We were able to observe the progress of our case in detail because we had followed up for 8 years.

\section{Conclusion}

Although a cystic change of PNET is generally caused by ischemia or necrosis inside the tumor, some PNETs may occur as a small cyst and increase without changing form.

\section{Abbreviations}

PNET: Pancreatic neuroendocrine tumor; CT: Computed tomography; ERP: Endoscopic retrograde pancreatography; CEA: Carcinoembryonic antigen; CA19-9: Carbohydrate antigen 19-9; CECT: Contrast-enhanced CT MRI: Magnetic resonance imaging

\section{Acknowledgements}

Not applicable.

\section{Presentation}

We have not presented this article to date.

\section{Authors' contributions}

KN described and designed the article. TK edited the article. All authors approved the final manuscript.

\section{Funding}

This study was not funded.

\section{Availability of data and materials}

All data regarding this paper are included in this published article.

\section{Ethics approval and consent to participate}

This study was conducted in accordance with the ethical standards of our institute.

\section{Consent for publication}

Written informed consent was obtained from the patient for publication of this case.

\section{Competing interests}

The authors declare that they have no conflicts of interest.

\section{Author details}

'Department of Surgery, National Hospital Organization Nagasaki Medical Center, 2-1001-1, Kubara, Omura City, Nagasaki 856-8562, Japan. ${ }^{2}$ Department of Pathology, National Hospital Organization Nagasaki Medical Center, Omura, Japan.

Received: 27 May 2020 Accepted: 19 June 2020

Published online: 29 June 2020

\section{References}

1. Kongkam P, Al-Haddad M, Attasaranya S, O'Neil J, Pais S, Sherman S, et al. EUS and clinical characteristics of cystic pancreatic neuroendocrine tumors. Endoscopy. 2008:40(7):602-5.

2. Bordeianou L, Vagefi PA, Sahani D, Deshpande V, Rakhlin E, Warshaw AL, et al. Cystic pancreatic endocrine neoplasms: a distinct tumor type? J Am Coll Surg. 2008;206(6):1154-8

3. Caglia P, Cannizzaro MT, Tracia A, Amodeo L, Tracia L, Buffone A, et al. Cystic pancreatic neuroendocrine tumors: to date a diagnostic challenge. Int J Surg. 2015;21(Suppl 1):S44-9.

4. Singhi AD, Chu LC, Tatsas AD, Shi C, Ellison TA, Fishman EK, et al. Cystic pancreatic neuroendocrine tumors: a clinicopathologic study. Am J Surg Pathol. 2012;36(11):1666-73.

5. Nakashima Y, Ohtsuka T, Nakamura S, Mori Y, Nakata K, Miyasaka Y, et al. Clinicopathological characteristics of non-functioning cystic pancreatic neuroendocrine tumors. Pancreatology. 2019;19(1):50-6.
6. Koh YX, Chok AY, Zheng HL, Tan CS, Goh BK. A systematic review and meta-analysis of the clinicopathologic characteristics of cystic versus solid pancreatic neuroendocrine neoplasms. Surgery. 2014;156(1):83-96 e2.

7. Thorlacius H, Kalaitzakis E, Johansson GW, Ljungberg O, Ekberg O, Toth E. Cystic neuroendocrine tumor in the pancreas detected by endoscopic ultrasound and fine-needle aspiration: a case report. BMC Res Notes. 2014;7: 510.

8. Baker MS, Knuth JL, DeWitt J, LeBlanc J, Cramer H, Howard TJ, et al. Pancreatic cystic neuroendocrine tumors: preoperative diagnosis with endoscopic ultrasound and fine-needle immunocytology. J Gastrointest Surg. 2008:12(3):450-6.

9. Goh B, Ooi L, Tan YM, Cheow P, Chung Y, Chow P, et al. Clinicopathological features of cystic pancreatic endocrine neoplasms and a comparison with their solid counterparts. Eur J Surg Oncol. 2006;32:553-6.

10. Ahrendt SA, Komorowski RA, Demeure MJ, Wilson SD, Pitt HA. Cystic pancreatic neuroendocrine tumors: is preoperative diagnosis possible? J Gastrointest Surg. 2002;6(1):66-74.

11. Kamisawa T, Fukayama M, Koike M, Tabata I, Okamoto A. A case of malignant cystic endocrine tumor of the pancreas. Am J Gastroenterol. 1987;82(1):86-9.

12. Takeshita K, Furui S, Makita K, Yamauchi T, Irie T, Tsuchiya K, et al. Cystic islet cell tumors: radiologic findings in three cases. Abdom Imaging. 1994;19(3): $225-8$.

\section{Publisher's Note}

Springer Nature remains neutral with regard to jurisdictional claims in published maps and institutional affiliations.

\section{Submit your manuscript to a SpringerOpen ${ }^{\circ}$ journal and benefit from:}

- Convenient online submission

- Rigorous peer review

- Open access: articles freely available online

- High visibility within the field

- Retaining the copyright to your article

Submit your next manuscript at $\boldsymbol{\nabla}$ springeropen.com 